\title{
KRONECKER FUNCTION RINGS AND FLAT $D[X]$-MODULES ${ }^{1}$
}

\section{J. T. ARNOLD AND J. W. BREWER}

AbSTRACr. Let $D$ be an integral domain with identity. Gilmer has recently shown that in order that a $v$-domain $D$ be a Prüfer $v$-multiplication ring, it is necessary and sufficient that $D^{v}$ be a quotient ring of $D[X]$, where $D^{v}$ is the Kronecker function ring of $D$ with respect to the $v$-operation. In this paper the authors prove that in the above theorem it is possible to replace "a quotient ring of $D[X]$ " with "a flat $D[X]$-module." Moreover, it is shown that $D^{v}$ is the only Kronecker function ring of $D[X]$ which can ever be a flat $D[X]$-module.

In the sequel $D$ will denote an integral domain with identity and $K$ will denote its quotient field. Otherwise, our notation is essentially that of [1].

Let $I(D)$ denote the collection of all fractional ideals of $D$. The mapping $F \rightarrow F_{v}$ of $I(D)$ into $I(D)$, where $F_{v}=\left(F^{-1}\right)^{-1}$, is called the $v$ operation on $D$. The $v$-operation satisfies the properties of a *-operation, and if the $v$-operation is endlich arithmetisch brauchbar, then we call $D$ a $v$-domain and we denote by $D^{v}$ the Kronecker function ring of $D$ with respect to the $v$-operation (for a detailed treatment of *-operations, Kronecker function rings and the $v$-operation, see [1, Chapters 26 and 28]). If the set of $v$-ideals of finite type is a group under $v$-multiplication, then $D$ is said to be a Prüfer $v$-mulliplication ring. Let $D$ be a $v$-domain. Then in [2] Gilmer proves that $D$ is a Prüfer $v$-multiplication ring if and only if $D^{v}$ is a quotient ring of $D[X]$. Thus, in case $D$ is a Prüfer $v$-multiplication ring, $D^{v}$ is a flat $D[X]$-module. The converse is also true, but in order to prove it we require some preliminary results.

LEMMA 1. Let $D$ be an integral domain. If $Q$ is a prime ideal of $D[X]$ such that $(D[X])_{Q}$ is a valuation ring and if $(Q \cap D) D[X] \subset Q$, then $Q \cap D=(0)$.

Presented to the Society, November 20, 1970 under the title Essential valuation overrings of $D[X]$; received by the editors May 18, 1970 and, in revised form, July 15, 1970.

AMS 1969 subject classifications. Primary 1393; Secondary 1398.

Key words and phrases. Kronecker function ring, flat module, essential valuation ring, Prüfer $v$-multiplication ring.

1 The authors are indebted to the referee for several helpful suggestions concerning the paper. 
Proof. Since $D_{Q \cap D}=(D[X])_{Q} \cap K$, there is no loss of generality in assuming that $D$ is a valuation ring and that $Q \cap D$ is its maximal ideal. $Q$ is generated $\bmod (Q \cap D) D[X]$ by a monic polynomial $f$. If $y \in Q \cap D$, then since $(D[X])_{Q}$ is a valuation ring and since $f \notin$ $(Q \cap D)(D[X])_{Q}$, it follows that $y=f g / h$, where $g \in D[X]$ and $h \in$ $D[X]-Q$. If $y \neq 0$, then $f$ divides $h$ in $K[X]$, whence $f$ divides $h$ in $D[X]$ by $[1,8.4]$.

The following result, due to Richman [3, Theorem 2], will be of use.

Lemma 2. Let $D_{1}$ be an overring of $D$-that is, $D \subseteq D_{1} \subseteq K$. In order that $D_{1}$ be a flat $D$-module it is necessary and sufficient that $\left(D_{1}\right)_{M_{1}}$ $=D_{M_{1} \cap D}$ for each maximal ideal $M_{1}$ of $D_{1}$.

We are now able to sharpen the aforementioned result of Gilmer.

Theorem 3. Let $D$ be a v-domain and let $D^{v}$ be the Kronecker function ring of $D$ with respect to the v-operation. The following conditions are equivalent:

(1) $D$ is a Prïfer v-multiplication ring.

(2) $D^{v}$ is a quotient ring of $D[X]$.

(3) Each valuation overring of $D^{v}$ is of the form $(D[X])_{P[X]}$ where $D_{P}$ is a valuation overring of $D$.

(4) $D^{v}$ is a flat $D[X]$-module.

Proof. The equivalence of (1) and (2) is given in [2]. That (2) implies (3) is a direct consequence of Lemma 1 and that (3) implies (4) follows from Lemma 2. Therefore, we need only show that (4) implies (2). We claim that $D^{v}=(D[X])_{S}$, where $S=\left\{f \in D[X] \mid\left(A_{f}\right)_{\text {。 }}\right.$ $=D\}$. (Here, $A_{f}$ denotes the ideal of $D$ generated by the coefficients of $f$.) Clearly, $D^{v} \supseteq(D[X])_{s}$. Let $A$ be an ideal of $D[X]$ such that $A D^{v}=D^{v}$. Then there exist $f_{1}, \cdots, f_{n} \in A$ such that $\left(f_{1}, \cdots, f_{n}\right) D^{v}$ $=D^{v}$. Set $m=\max _{1 \leqq i \leqq n}\left\{\operatorname{deg}\left(f_{i}\right)\right\}+1$ and put $f(X)=f_{1}+f_{2} X^{m}$ $+\cdots+f_{n} X^{(n-1) m}$. Then by $[1,26.7],\left(A_{f}\right)_{v}=D$ and hence $A \cap S$ $\neq \varnothing$. Therefore, if $M$ is a maximal ideal of $(D[X])_{S}$, then $M D^{v} \subset D^{v}$ and there exists a maximal ideal $M^{\prime}$ of $D^{v}$ such that $M=M^{\prime} \cap(D[X])_{S}$. The result follows from Lemma 2.

On the basis of Theorem 3, one is led to ask what Kronecker function rings are flat $D[X]$-modules. The answer is given by

CoROllary 4. If $D^{\prime}$ is a Kronecker function ring of $D$ which is a flat $D[X]$-module, then $D^{\prime}=D^{v}$.

Proof. It follows from Lemma 1 and Lemma 2 that each valuation overring of $D^{\prime}$ is of the form $(D[X])_{P_{\alpha}[X]}$, where $D_{P_{\alpha}}$ is a valuation 
overring of $D$. Therefore, $D=D^{\prime} \cap K=\left(\bigcap_{\alpha}(D[X])_{P_{\alpha}[X]}\right) \cap K=\bigcap_{\alpha} D_{P_{\alpha}}$ and it follows from $[1,36.13]$ that $D^{\prime}=D^{v}$.

\section{REFERENCES}

1. R. Gilmer, Multiplicative ideal theory, Queen's Papers in Pure and Appl. Math., no. 12, Queen's University, Kingston, Ontario, 1968. MR 37 \#5198.

2. —- An embedding theorem for HCF rings, Proc. Cambridge Philos. Soc. 68 (1970), 583-587.

3. F. Richman, Generalized quotient rings, Proc. Amer. Math. Soc. 16 (1965), 794799. MR 31 \#5880.

Virginia Polytechnic Institute and State University, Blacksburg, VIRGINIA 24061 